\title{
Photonic Topological Dissipation in Time-Multiplexed Resonator Networks
}

\author{
Christian Leefmans ${ }^{1}$, Avik Dutt ${ }^{3}$, James Williams ${ }^{2}$, Luqi Yuan ${ }^{4}$, Midya Parto ${ }^{2}$, \\ Franco Nori ${ }^{5,6}$, Shanhui Fan ${ }^{3}$, Alireza Marandi ${ }^{1,2}$ \\ ${ }^{1}$ Department of Applied Physics, California Institute of Technology. 1200 E California Blvd., Pasadena, CA \\ 91125, USA \\ ${ }^{2}$ Department of Electrical Engineering, California Institute of Technology. 1200 E California Blvd., Pasadena, \\ CA 91125, USA. \\ ${ }^{3}$ Department of Electrical Engineering, Stanford University, Stanford, CA 94305, USA. \\ ${ }^{4}$ School of Physics and Astronomy, Shanghai Jiao Tong University, Shanghai 200240, China \\ ${ }^{5}$ Theoretical Quantum Physics Laboratory, RIKEN Cluster for Pioneering Research, Wako-shi, Saitama \\ 351-0198, Japan \\ ${ }^{6}$ Department of Physics, University of Michigan, Ann Arbor, Michigan 48109-1040, USA \\ marandi@caltech.edu
}

\begin{abstract}
We utilize dissipatively coupled, time-multiplexed photonic resonator networks to demonstrate topologically nontrivial behaviors in the dissipation of one- and twodimensional lattices. We present edge state and band structure measurements of the these networks. (c) 2021 The Author(s)
\end{abstract}

For over a decade, topological photonics has focused on nontrivial topology in optical systems with conservatively coupled elements [1]. However, driven dissipative systems also possess intruiging behaviors and potential applications in both the quantum and classical regimes [2,3]. With regards to topology, dissipative systems enable time-reversal symmetry breaking interactions and provide new methods of achieving nonzero topological invariants [4].

Here we introduce the notion of photonic topological dissipation: By coupling the nodes of a time-multiplexed resonator network through Lindbladian, systemreservoir interactions, we induce nonzero topological invariants in the dissipation spectra (state-dependent dissipation) of our network. Fig. 1 illustrates the differences between our open, dissipative system and the more familiar closed, Hamiltonian systems, which can exhibit topologically nontrivial frequency spectra.

We demonstrate photonic topological dissipation by experimentally realizing the SSH and Harper-Hofstadter models with the dissipative time-multiplexed resonator network shown in Fig. 2(a). The delay lines of this network can implement nearestneighbor hopping along a 1D chain or along both directions of a synthetic square

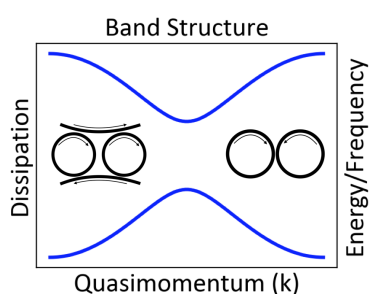

Fig. 1: Conservative and Dissipative Coupling Dissipatively coupled systems are characterized by a dissipation spectrum, while conservatively coupled systems are characterized by a frequency spectrum. lattice. The delay line intensity modulators, $\mathrm{IM}_{ \pm 1}$, introduce the staggered coupling strengths of the SSH model, while the delay line phase modulators, $\mathrm{PM}_{ \pm 4}$, emulate the gauge field of the HarperHofstadter model. Furthermore, $\mathrm{IM}_{ \pm 1}$ and $\mathrm{IM}_{C}$ enable us to impose both periodic boundary conditions (PBCs) and open boundary conditions (OBCs) on our lattices.

We probe the topological behaviors of these models by using $\mathrm{IM}_{0}$ and $\mathrm{PM}_{0}$ [Fig. 2(a)] to inject their eigenstates into the network. When the couplings of the network correspond to those of the model, the eigenstates resonate in the network. Otherwise, they do not. We use this procedure to extract the SSH band structure at different parameter regimes [Fig. 2(b)-(c)] and to observe a dynamical phase transition between the model's trivial and topological states [Fig. 2(d)-(f)]. We then measure a topological edge state of the Harper-Hofstadter model [Fig. 3(a)-(c)].

In summary, we have shown that a dissipatively coupled network of resonators can exhibit topologically nontrivial behaviors in its state-dependent dissipation. We have experimentally demonstrated some of these behaviors in $1 \mathrm{D}$ and $2 \mathrm{D}$ lattices in the same time-multiplexed network. The results can open new opportunities for optical driven dissipative systems in the classical and quantum regimes.

The authors gratefully ackowledge the support of ARO Grant No. W911NF-18-1-0285, NSF Grants No. 1846273 and 1918549, and NTT Research Inc.

\section{References}

1. T. Ozawa, et al., “Topological photonics,” Rev. Mod. Phys. 91, 015006 (2019).

2. C.-E. Bardyn et al., “Topology by dissipation,” New J. Phys. 15, 085001 (2013). 
3. A. Marandi et al., "Network of time-multiplexed optical parametric oscillators as a coherent ising machine," Nat. Photonics. 8, 937 (2014).

4. C. Wanjura et al., "Topological framework for directional amplification in driven-dissipative cavity arrays," Nat. Comm. 11, 3149 (2020).

(a)

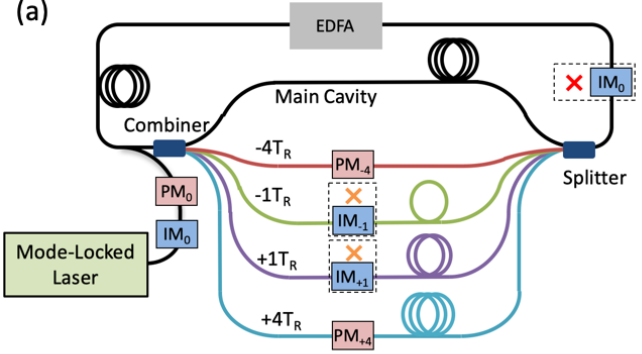

(b)

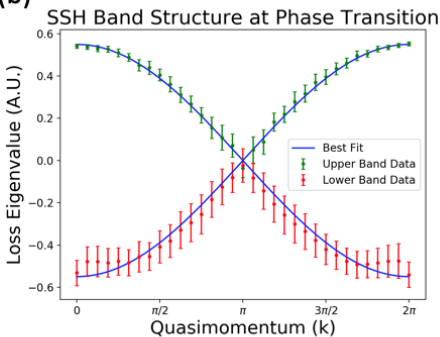

(c)

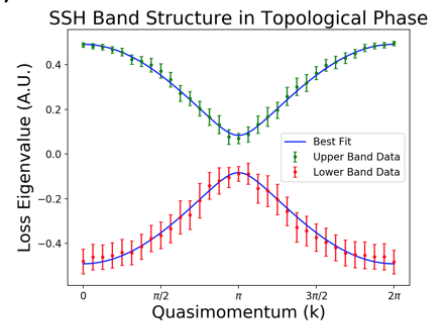

(d)

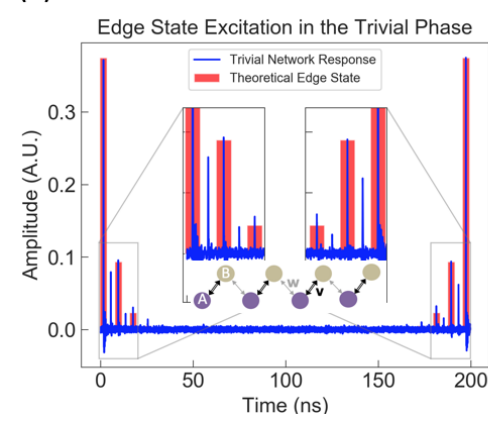

(e)

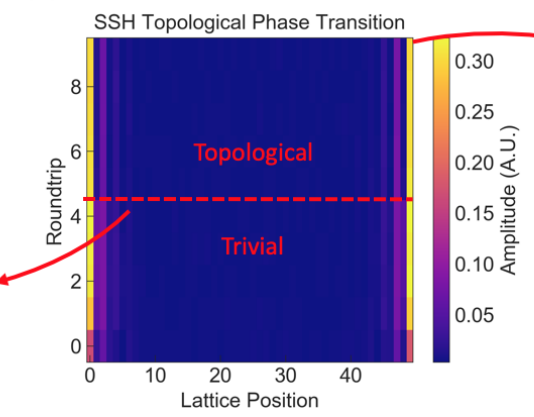

(f)

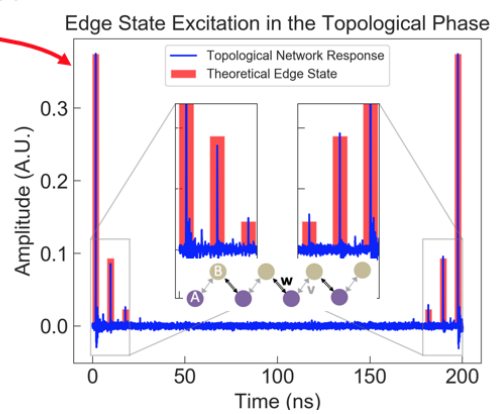

Fig. 2: SSH Band Structure and Phase Transition (a) We realize the SSH model and the Harper-Hofstadter model in a dissipatively coupled, time-multiplexed resonator network with four delay lines. To study the SSH model, we block the $\pm 4 T_{R}$ delay lines, and we program the intensity modulators, $\mathrm{IM}_{ \pm 1}$ to implement the staggered couplings of the SSH model $\left(H=\sum_{n_{x}} v \hat{a}_{n_{x}}^{\dagger} \hat{b}_{n_{x}}+w \hat{a}_{n_{x}}^{\dagger} \hat{b}_{n_{x}+1}+\right.$ h.c. $)$. We inject the Bloch wave eigenstates of the SSH model into the network and measure their resonant amplitudes to construct the dissipation spectra of the SSH network (b) at the phase transition point $(w / v=1)$ and (c) in the topological phase $(w / v=\sqrt{2})$.(e) We observe a dynamical phase transition by injecting the SSH edge state corresponding to $w / v=2$ into the network. (d) With the network couplings in the trivial phase $(w / v=1 / 2)$, we observe that the edge state does not remain localized. (f) When we switch the network couplings to the topological phase $(w / v=2)$, we observe the localized edge state.
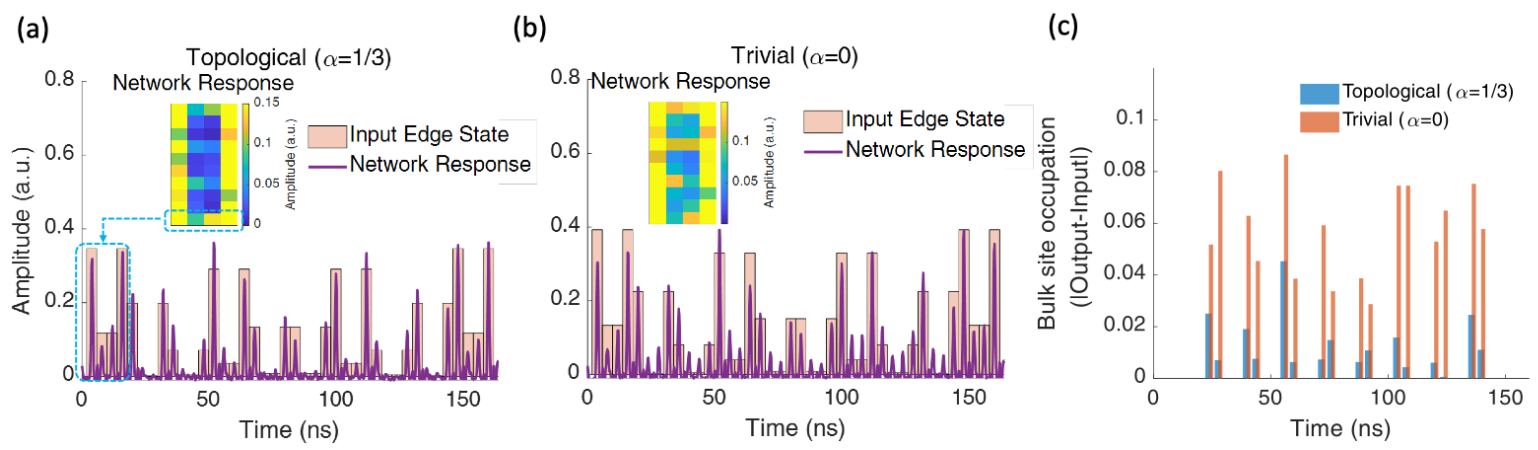

Fig. 3: Harper-Hofstadter Edge State (a) We program the couplings of the network to simulate the Harper-Hofstadter model $\left(H=J \sum_{n_{x}, n_{y}} \hat{a}_{n_{x}+1, n_{y}}^{\dagger} \hat{a}_{n_{x}, n_{y}}+\hat{a}_{n_{x}, n_{y}+1}^{\dagger} \hat{a}_{n_{x}, n_{y}} e^{i 2 \pi \alpha n_{x}}+\right.$ h.c. $)$ with an effective magnetic field of $\alpha=1 / 3$ and OBCs. The edge state remains localized in the injected state. (b) With no gauge field $(\alpha=0)$, the network implements a trivial tight-binding model. In this case, and the state diffuses into the bulk. (c) The bulk occupation of the resonant states in the topological and trivial lattices confirms the localization of the topological edge state. (Note: the color bars in (a) and (b) are saturated to emphasize the bulk sites.) 